\title{
Bilateral retinal venous occlusion in Fabry disease
}

\author{
Simon Dulz ${ }^{1 *}$, Yevgeniya Atiskova ${ }^{1}$, Anja Friederike Köhn² ${ }^{2}$ Nicole Muschol ${ }^{2}$ \\ ${ }^{1}$ Department of Ophthalmology, University Medical Center Hamburg-Eppendorf \\ ${ }^{2}$ Department of Pediatrics, University Medical Center Hamburg-Eppendorf
}

Received: 23 July, 2016; Accepted: 04 August, 2016; Published: 14 August, 2016

*Corresponding author: Simon Dulz, Department of Ophthalmology, University Medical Center Hamburg-Eppendorf; Tel. no: 0160-7971262; E-mail: s.dulz@uke.de

Fabry disease is an X-linked lysosomal storage disorder. Due to a deficiency of alpha galactosidase A (alpha Gal A) there is adeposition of sphingo lipids within the vascular endothelium. This is associated with vascular dysfunction, alteration in vascular perfusion and a prothromboticstate [1]. Here we report of a 21 year-old male patient with acute or chronic renal failure. Kidney biopsy revealed storage material consistent with Fabry disease. Diagnosis was confirmed by deficient alpha-Gal A-activity. Genetic test in greveiled a previously undescribed homozygous mutation (Exon 5: c.689_697del9pb (p.Ala230_Ile232del) in the GLA gene. The patient started hemodialysis as well as enzyme replacement therapy with a galsidase beta (Fabrazyme; Genzyme).Two months later the patient was admitted to hospital due to an acute of myocardial insufficiency with pericardial effusion. Additionally at admission to the hospital the patient reported of a predominantly left-sided visual impairment.

At ophthalmic exam, best-corrected visual acuity at 1 meter was 20/63 in the right eye and 1/40 in the left eye. The anterior eye segment examination revealed bilateral conjunctival vessel tortuositas in all four quadrants as well as a bilateral grade 1 cornea verticillata. A clear lens status and an otherwise quite anterior chamber were recorded. Fundus examination exhibited a bilateral optic disc edema with panretinal flame-shaped retinal hemorrhages, peripheral cotton wool spots and a macular edema with ring-shaped extra foveal hard exudates on the left side. (Figure 1) An Optical Coherence Tomography (OCT) examination (Heidelberg Spectral is; Heidelberg Engineering) exposed a leftsided cystoid macular edema (Figure 2) and a right-sided regular foveal impression (not shown) besides bilateral parapapillary subretinal fluid accumulation. We were not able to perform a fundus angiography due to the terminal renal failure and the necessity to perform daily hemodialysis as well as the reduced general health of the patient.

We proposed the diagnosis of a right-sided partial and a left side complete Central Retinal Venous Occlusion (CRVO) and started a monthly intravitreal therapy regime with Bevacizumab (Avastin; Roche) in the left eye.

Ocular manifestations of Fabry disease are cornea verticillata, a whorl-like corneal pattern, cataract, as well as conjunctival and retinal vessel tortuositas. Retinal vascular tortuous it as is present in approximately $20 \%$ of patients with Fabry disease and correlates well with disease severity [2, 3]. Ocular complications have rarely been described. Single case reports on retinal arterial occlusion in one patient as well as a case of retinal venous occlusion have been published so far $[4,5]$.

An increased risk for systemic thromboembolic complications including an increased stroke risk have been described in Fabry disease [6]. Nevertheless, ocular thromboembolic events are rather uncommon, but a sight-threatening complication. This case of a bilateral CRVO in young men with Fabry disease is most likely due to fabry specific retinal vascular abnormalities and highlights Fabry disease as a differential diagnosis of juvenile and presenile CRVO.

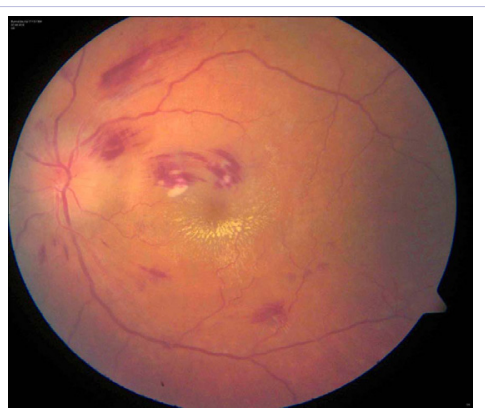

Figure 1: Fundus photography (left eye) - Optic disc edema with parapaillar flame-shaped retinal hemorraghes extending along the vascular arches and parafoveal hard exudates.

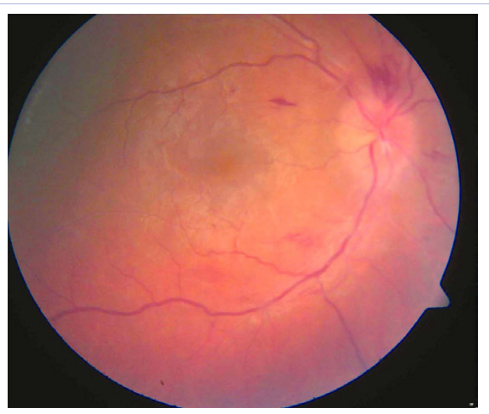

Figure 2: Fundus photography (right eye) - Optic disc edema with vessel narrowing and superior parapapillar flame-shaped haemorrhages. 


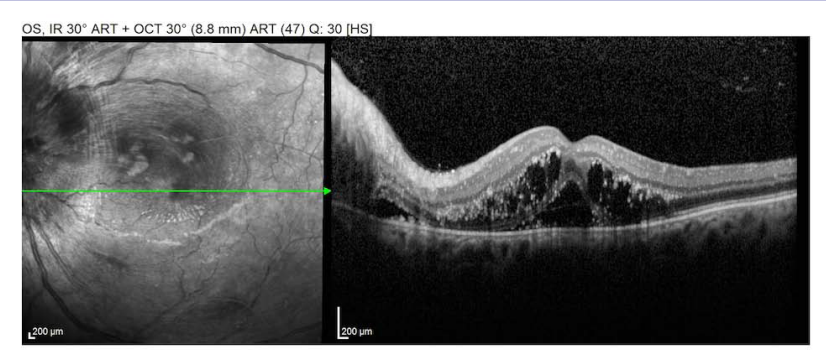

Figure 3: OCT imaging (left eye) - Intraretinal cystoid macular edema along with subfoveal fluid accumulation.

\section{References}

1. Moore DF, Kaneski CR, Askar H, Schiffmann R. The cerebral vasculopathy of Fabry disease. J Neurol Sci. 2007;257(1-2):258-263.

2. Fledelius HC, Sandfeld L, Rasmussen ÅK, Madsen CV, Feldt-Rasmussen U. Ophthalmic experience over 10 years in an observational nation wide Danish cohort of Fabry patients with access to enzyme replacement. Acta Ophthalmol. 2015;93(3):258-264. doi: 10.1111/ aos.12588.

3. Pitz S, Kalkum G, Arash L, Karabul N, Sodi A, Larroque S, Beck M, Gal A. Ocular signs correlate well with disease severity and genotype in Fabry disease. PLoS One. 2015;10(3):e0120814. doi: 10.1371/ journal.pone.0120814.

4. Oto S, Kart H, Kadayifçilar S, Ozdemir N, Aydin P. Retinal vein occlusion in a woman with heterozygous Fabry's disease. Eur J Ophthalmol. 1998;8(4):265-267.

5. Mitchell KT, Bradley JC, Gilmore LS, Meyerrose GE. Sequential bilateral central retinal artery occlusions in a female carrier of Fabry disease. Clin Experiment Ophthalmol. 2009 37(7):748-50. doi: 10.1111/j.1442-9071.2009.02124.x.

6. Utsumi K, Yamamoto N, Kase R, Takata T, Okumiya T, Saito H, Suzuki T, Uyama E, Sakuraba H. High incidence of thrombosis in Fabry's disease. Intern Med. 1997;36(5):327-329. 